\title{
“HCR's Series"
}

\section{Mr Harish Chandra Rajpoot}

Madan Mohan Malaviya University of Technology, Gorakhpur-273010 (UP) India

\begin{abstract}
This is a finite series which is the sum of first ' $n$ ' natural numbers multiplied by their own respective factorials. The series has been derived from HCR's Rank formula which was proposed by the author. It is extremely useful in case studies \& computations. Although HCR's Series is different from the Arithmetic, Geometric, Harmonic \& Taylor's Series of simple functions, it is the expansion of factorial of any natural number in form of discrete summation thus it is also named as HCR's divergence series.
\end{abstract}

\section{Derivation of HCR's Series:}

Let there be $(n+1)$ articles which are non-repetitive \& have a pre-defined linear sequence according to their priority as follows

$$
A_{1}, A_{2}, A_{3}, A_{4}, A_{5} \ldots \ldots \ldots \ldots \ldots \ldots A_{n-3}, A_{n-2}, A_{n-1}, A_{n}, A_{n+1}
$$

In this case the total number of all possible linear arrangements obtained by permuting all these $(n+1)$ articles together is given as follows

$$
N_{t}=(n+1) \times n \times(n-1) \times(n-2) \ldots \ldots \ldots \ldots \ldots \ldots . . \ldots \times 4 \times 3 \times 2 \times 1=(n+1) !
$$

If all these linear arrangements obtained are arranged in the correct order according to the same priority then the last linear permutation in the arrangements will be as follows

$$
A_{n+1} A_{n} A_{n-1} A_{n-2} A_{n-3} \ldots \ldots \ldots \ldots \ldots \ldots A_{5} A_{4} A_{3} A_{2} A_{1}
$$

Since, all $N_{t}=(n+1)$ ! permutations are arranged in correct order then the rank (position) of the last linear permutation must be equal to the total number of the linear permutations

Now, rank of last linear permutation (using HCR's Rank Formula discovered by the author) is given as follows

$$
\begin{aligned}
\therefore & N_{t}=R\left(A_{n+1} A_{n} A_{n-1} A_{n-2} A_{n-3} \ldots \ldots \ldots \ldots \ldots . . . \ldots A_{5} A_{4} A_{3} A_{2} A_{1}\right)=\sum_{i=1}^{i=n+1} F_{i}\left(\frac{P_{i}}{S_{i}}\right) \\
\Rightarrow(n+1) !=F_{1} & \left(\frac{P_{1}}{S_{1}}\right)+F_{2}\left(\frac{P_{2}}{S_{2}}\right)+F_{3}\left(\frac{P_{3}}{S_{3}}\right)+F_{4}\left(\frac{P_{4}}{S_{4}}\right)+\ldots \ldots \ldots \ldots \ldots+F_{n-2}\left(\frac{P_{n-2}}{S_{n-2}}\right)+F_{n-1}\left(\frac{P_{n-1}}{S_{n-1}}\right)+F_{n}\left(\frac{P_{n}}{S_{n}}\right) \\
& +F_{n+1}\left(\frac{P_{n+1}}{S_{n+1}}\right)
\end{aligned}
$$

Where $F \rightarrow$ Formerity, $P \rightarrow$ Permuty \& $S \rightarrow$ Similarity

$F=$ number of articles appearing before a selected one in correct linear sequence

$P=$ number of linear permutations obtained by the articles excluding the selected one $S=$ number of articles similar to the selected one including it

But all the articles are non-repetitive (dissimilar) hence

$$
\begin{gathered}
S_{1}=S_{2}=S_{3}=S_{4}=S_{5}=\ldots \ldots \ldots \ldots=S_{n-2}=S_{n-1}=S_{n}=S_{n+1}=1 \\
\therefore(n+1) !=F_{1} P_{1}+F_{2} P_{2}+F_{3} P_{3}+F_{4} P_{4}+\ldots \ldots \ldots \ldots+F_{n-2} P_{n-2}+F_{n-1} P_{n-1}+F_{n} P_{n}+F_{n+1} P_{n+1}
\end{gathered}
$$

Now, let's find the values of parameters F \& P corresponding to each of $(n+1)$ articles, using Method of Cancellation as follows

According to last linear permutation $A_{n+1} A_{n} A_{n-1} A_{n-2} A_{n-3} \ldots \ldots \ldots \ldots \ldots A_{5} A_{4} A_{3} A_{2} A_{1}$, starting from the left, the first article is $A_{n+1}$ Hence, select \& label (*) this article in the pre-defined linear sequence of articles as follows

$$
A_{1}, A_{2}, A_{3}, A_{4}, A_{5} \ldots \ldots \ldots \ldots \ldots \ldots A_{n-3}, A_{n-2}, A_{n-1}, A_{n}, A_{n+1} *
$$

Now, $F_{1}=$ number of articles appearing before labelled $A_{n+1}$ in above sequence $=n$

$$
P_{1}=\text { linear permutations of articles excluding } A_{n+1} \text { in above sequence }=n !
$$

Now, remove (cancel) this article $A_{n+1}$ (labelled by (*)), now linear sequence of remaining articles is as follows

$$
A_{1}, A_{2}, A_{3}, A_{4}, A_{5} \ldots \ldots \ldots \ldots \ldots \ldots A_{n-3}, A_{n-2}, A_{n-1}, A_{n}
$$

According to last linear permutation $A_{n+1} A_{n} A_{n-1} A_{n-2} A_{n-3} \ldots \ldots \ldots \ldots \ldots . . . A_{5} A_{4} A_{3} A_{2} A_{1}$ the next article is $A_{n}$ Hence, select \& label (*) this article in the above linear sequence of articles as follows

$$
A_{1}, A_{2}, A_{3}, A_{4}, A_{5} \ldots \ldots \ldots \ldots \ldots \ldots A_{n-3}, A_{n-2}, A_{n-1}, A_{n} *
$$

Now, $F_{2}=$ number of articles appearing before labelled $A_{n}$ in above sequence $=n-1$ 
$P_{2}=$ linear permutations of articles excluding $A_{n}$ in above sequence $=(n-1)$ !

Now, remove (cancel) this article $A_{n}$ (labelled by (*)), now linear sequence of remaining articles is as follows

$$
A_{1}, A_{2}, A_{3}, A_{4}, A_{5} \ldots \ldots \ldots \ldots \ldots \ldots . A_{n-3}, A_{n-2}, A_{n-1}
$$

According to last linear permutation $A_{n+1} A_{n} A_{n-1} A_{n-2} A_{n-3} \ldots \ldots \ldots \ldots \ldots \ldots A_{5} A_{4} A_{3} A_{2} A_{1}$ the next article is $A_{n-1}$ Hence, select \& label (*) this article in the above linear sequence of articles as follows

$$
A_{1}, A_{2}, A_{3}, A_{4}, A_{5} \ldots \ldots \ldots \ldots \ldots \ldots A_{n-3}, A_{n-2}, A_{n-1} *
$$

Now, $F_{3}=$ number of articles appearing before labelled $A_{n-1}$ in above sequence $=n-2$

$$
P_{3}=\text { linear permutations of articles excluding } A_{n-1} \text { in above sequence }=(n-2) \text { ! }
$$

Now, remove (cancel) this article $A_{n-1}$ (labelled by (*)), make new sequence then select $\&$ label next article in the new sequence to find its parametric values $F_{4} \& P_{4}$

Repeat this process until reaches last article $A_{1}$ of the last permutation $A_{n+1} A_{n} A_{n-1} A_{n-2} A_{n-3} \ldots \ldots A_{4} A_{3} A_{2} A_{1}$ for which $F_{n+1}=1 \& P_{n+1}=1$ thus we find parametric values of all $(n+1)$ articles as follows

$\left(F_{1}=n, P_{1}=n !\right),\left(F_{2}=(n-1), P_{2}=(n-1) !\right),\left(F_{3}=(n-2), P_{3}=(n-2) !\right)$,

$\left(F_{4}=(n-3), P_{4}=(n-3) !\right),\left(F_{5}=(n-4), P_{5}=(n-4) !\right), \ldots \ldots \ldots \ldots \ldots \ldots,\left(F_{n-2}=3, P_{n-2}=3 !\right)$,

$\left(F_{n-1}=2, P_{n-1}=2 !\right),\left(F_{n}=1, P_{n}=1 !\right),\left(F_{n+1}=1, P_{n+1}=1\right)$,

Now on setting the values of all the parameters as follows, we get

$$
\begin{aligned}
\Rightarrow(n+1) !=n \times & n !+(n-1) \times(n-1) !+(n-2) \times(n-2) !+(n-3) \times(n-3) !+\ldots \ldots \ldots \ldots+4 \times 4 ! \\
& +3 \times 3 !+2 \times 2 !+1 \times 1 !+1 \times 1 \\
=(1 \times 1 !+2 \times 2 & 2 !+3 \times 3 !+4 \times 4 !+\ldots \ldots \ldots \ldots \ldots \ldots \ldots \ldots \ldots+(n-3) \times(n-3) !+(n-2) \times(n-2) ! \\
& +(n-1) \times(n-1) !+n \times n !)+1
\end{aligned}
$$

Using discrete summation, we have

$$
\begin{gathered}
\Rightarrow(n+1) !=\sum_{i=1}^{i=n}[i \times i !]+1 \\
\sum_{i=1}^{i=n}[i \times i !]=(n+1) !-1
\end{gathered}
$$

\section{Verification of HCR's Series: Using 'Mathematical Induction'}

On setting $n=1$ in the expression (1), we get

$$
\begin{gathered}
\sum_{i=1}^{i=1}[i \times i !]=(1+1) !-1 \\
\Rightarrow 1 \times 1 !=2 !-1 \text { or } 1=1
\end{gathered}
$$

The above expression (1) is true for $n=1$

Let's assume that the expression (1) is true for $n=k$ then we have

Or

$$
\sum_{i=1}^{i=k}[i \times i !]=(k+1) !-1
$$

$$
1 \times 1 !+2 \times 2 !+3 \times 3 !+4 \times 4 !+\ldots \ldots \ldots \ldots \ldots+(k-1) \times(k-1) !+k \times k !=(k+1) !-1
$$

Now, on setting $n=k+1$ in the expression (1), we get

$$
\begin{aligned}
& \sum_{i=1}^{i=k+1}[i \times i !]=((k+1)+1) !-1=(k+2) !-1
\end{aligned}
$$

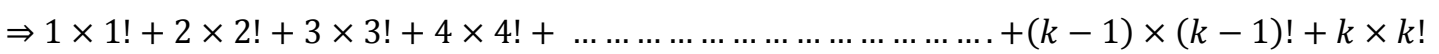

$$
\begin{aligned}
& +(k+1) \times(k+1) !=(k+2) !-1=(k+2) \times(k+1) !-1 \\
& \Rightarrow 1 \times 1 !+2 \times 2 !+3 \times 3 !+4 \times 4 !+\ldots \ldots \ldots \ldots \ldots \ldots \ldots+(k-1) \times(k-1) !+k \times k ! \\
& =(k+2) \times(k+1) !-1-(k+1) \times(k+1) !=(k+2-k-1) \times(k+1) !-1 \\
& \Rightarrow 1 \times 1 !+2 \times 2 !+3 \times 3 !+4 \times 4 !+\ldots \ldots \ldots \ldots \ldots+(k-1) \times(k-1) !+k \times k !=(k+1) !-1
\end{aligned}
$$

The above result is true from the expression (II), now we find that 
The expression ( 1 ) is true for $\mathrm{n}=1 \& \mathrm{n}=\mathrm{k}+1$ if it is true for $\mathrm{n}=\mathrm{k}$, thus by mathematical induction The expression (1) is true for all the positive integers (or natural numbers), hence we get

$$
\sum_{i=1}^{i=n}[i \times i !]=(n+1) !-1 \quad \forall n \in N
$$

The above series is called as “HCR's Series” of first 'n' natural numbers (positive integers).

\section{Expansion of Factorial of any Natural Number ' $n$ '}

On setting, $n=n-1$ in the above series, we have

$$
\begin{aligned}
& \sum_{i=1}^{i=n-1}[i \times i !]=((n-1)+1) !-1=n !-1 \\
& \Rightarrow n !=1+\sum_{i=1}^{i=n-1}[i \times i !]
\end{aligned}
$$

Above series is the expansion of any natural number, which can be used in computation \& simulation

\section{Conclusion:}

Thus, the series is an invention on the factorials of first ' $n$ ' natural numbers. The series sums up the finite terms that are similar to the arithmetic, geometric \& harmonic series. Moreover it is an expansion of the factorial of any natural number. This can be used in computations \& simulations to contract a series in to a single value (factorial) and to expand the factorial of any natural number depending on the type of problems of computations \& simulations. It can be extremely in case studies \& other academic purposes.

\section{Reference:}

[1]. The work of the author is original. It is an outcome of deep studies\& experiments carried out by the author on "HCR's Rank Formula" certified by IJMPSR

[2]. Manuscript ID: 004022014A (Website: www.researchpublish.com)Date: 19 Feb, 2014 